\title{
The Design of Simulation System for Magnetic Resonance Signal
}

\author{
Ying Liu, Xiao-Mei An \\ School of Medical Instrument and Food Engineering, \\ University of Shanghai for Science and Technology, \\ Shanghai, China \\ E-mail: ling2431@163.com, axm_usst@163.com
}

\begin{abstract}
Magnetic Resonance Imaging (MRI) technology has developed rapidly in recent years, and has been widely used in various fields, such as medicine, physics, chemistry and biology. The principles of MRI involve electromagnetism, nuclear physics, quantum mechanics and many other disciplines. In addition, the high price of MRI equipment and no open configuration information of commercial equipment make it difficult to learn for the professional students with a major in imaging or analysis detection, the relevant profession training in hospitals and companies, and scientific researching. A simulation system proposed in this thesis is developed to modeling and generating the magnetic resonance (MR) signal. Three basic sequences (Single-Pulse, SE and CPMG) of MR are analyzed by using MATLAB. The changes of the signal waveform can be observed by adjusting the parameters, and to better present the basic principles of MRI and influencing factors. The design can reduce the requirements of hardware and save the waiting time of the data acquisition effectively, so that MR technology can be widely applied to the laboratory teaching, scientific research and other fields.
\end{abstract}

Keywords-magnetic resonance; simulation system; FID

\section{INTRODUCTION}

Due to the rapid development of the medical industry, MRI technology is widely used in the major hospitals for its high contrast, arbitrary azimuth tomography, multi parameter imaging, nonionizing radiation and other advantages; at the same time, many universities also actively set up related courses to train professional talents. As a new technology, MRI technology is not easy for scientific research and daily teaching because of its short development time, expensive equipment and no open configuration information of commercial equipment. In this paper, an MR signal simulation system is proposed to enhance the understanding of the principles and students' interest in learning. This simulation system has a strong practicability in the experimental teaching training and scientific research for saving the waiting time of the data acquisition effectively.

\section{THE BASIS OF MRI}

Nuclear (mostly hydrogen nuclear) in the static magnetic field with Larmor precession frequency will not stop due to its spin properties. Putting a certain frequency of alternating magnetic field (RF field) on this nuclear spin system, then the nuclear spin system absorbs energy and transits to higher energy level when the magnetic field frequency equals to nuclear precession frequency. Removing the RF field, nuclear in an energized state releases part of the energy in the mode of electromagnetic wave absorbed before and go back to the lower energy. Carrying the relevant information of the object, electromagnetic waves are received by the relevant equipment and transmitted to image processing system for the follow-up processing. That's all for completed MRI process.

The MRI system consists of five subsystems: magnet subsystem, gradient subsystem, radio frequency (RF) subsystem, computer subsystem, and spectrometer subsystem. Magnet subsystem is to produce a main magnetic field which always be marked as $\mathrm{B}_{0}$, while gradient subsystem is used to generate and control the gradient of the magnetic field and provide the 3D information of the object. RF subsystem is to transmit RF pulse and receive the MR signal. Connecting the operator and the equipment is computer subsystem, which can undertake the parameter transmission, image reconstruction, storage and display. As central control system for MRI, spectrometer subsystem is responsible for the operation of the sequence generation and control.

The complex system and expensive equipment make it difficult for professional students and scientific researching. Applying an MR signal simulation system can not only be beneficial to study but reduce the requirements of hardware and save the waiting time of the data acquisition effectively.

\section{THE ANALYSIS OF BASIC SEQUENCE}

\section{A. Single-pulse Sequence}

The proton group in the static magnetic field $\left(\mathrm{B}_{0}\right)$ has the macroscopic magnetization vector (M) along the direction of the main magnetic field $(\mathrm{Z})$. After a specific frequency of 90 degree RF field $\left(B_{1}\right)$ applied vertically, the macroscopic magnetization vector turns over to the XOY plane with the direction of RF field as the axis. At the end of the RF pulse, the macroscopic magnetization vector is restored to the original direction $(\mathrm{Z})$, which is the relaxation process. The change of magnetization vector can be detected by the closed coil placed in vitro, and the FID signal is formed. After the $\mathrm{RF}$ pulse, a transverse magnetization vector $\left(\mathrm{M}_{\mathrm{XY}}\right)$ is obtained. Its motion process can be expressed by sin $\omega t$ or $\cos \omega t$, and the transverse magnetization vector will attenuate in the form of exponential. So the connection between the peak of the spin echo (SE) is according to the $\mathrm{e}^{(-\mathrm{t} T 2)}$ rule

The amplitude of the FID signal is related to the longitudinal magnetization vector $\left(\mathrm{M}_{0}\right)$, which is determined 
by the external magnetic field $\left(\mathrm{B}_{0}\right)$, and the signal changes in the form of sin $\omega t$ or $\cos \omega t$, so the formula of FID signal can be preliminary expressed as follows.

$$
\begin{gathered}
S=M_{0} \times \sin \omega t \times e^{-\frac{t}{T_{2}}}, \text { or } \\
S=M_{0} \times \cos \omega t \times e^{-\frac{t}{T_{2}}}
\end{gathered}
$$

In order to facilitate the research, cos $\omega t$ is used in this thesis and the initial value of the longitudinal magnetization vector is assumed as 1 , so the formula of FID signal can be expressed as follows.

$$
S=\cos \omega t \times e^{-\frac{t}{T_{2}}}
$$

Ignoring the impact of the inhomogeneous magnetic field, the attenuation of MR signal is mainly because that Larmor frequency $\left(\omega_{0}\right)$ is not equal to the frequency of RF field. By frequency mixing technique, we assume that $\omega$ is the difference between Larmor frequency and the frequency of RF field, so the formula of FID signal can be expressed as follows.

$$
S=\cos \left[0.001 \times\left(\gamma \times B_{0}-f\right) \times t\right] \times e^{-\frac{t}{T_{2}}}
$$

Among them, $\omega_{0}=\gamma \times \mathrm{B}_{0}$ represents Larmor frequency and $\gamma$ is the magnetic spin ratio of the hydrogen nucleus.

\section{B. SE Sequence}

SE sequence consists of a 90 degree pulse and a 180 degree pulse. A 90 degree pulse is applied first, half echo time $(\tau / 2)$ later a 180 degree pulse is applied. After one echo time $(\tau)$, the phase convergent function of 180 degree pulse is over and the signal reach the maximum value, so the peak of the SE signal is decreased firstly and then increased. The 180 degree pulse can eliminate the effect of magnetic field inhomogeneity. The attenuation of SE is related to the time parameter $\mathrm{T}_{2}{ }^{*}$. Signal acquisition time (T) can be expressed as $\mathrm{T}=\mathrm{TD}$ (signal sampling point) / SW (bandwidth). The range of independent variable time ( $t$ ) is set form $-T / 2$ to $T / 2$, and used its absolute value in the formula. Assume the initial value of the longitudinal magnetization vector is 1 , so the simulation formula of SE sequence can be expressed as follows.

$$
S=\cos \left[0.001 \times\left(\gamma \times B_{0}-f\right) \times t\right] \times e^{-\frac{|t| t}{T_{2}}}
$$

\section{CPMG Sequence}

Based on SE sequence, CPMG sequence consists of one 90 degree pulse and several 180 degree pulses. Several 180 degree pulses make the phase of transverse magnetization vector converge. Then several echoes are obtained, and the signal amplitude continues to decrease over time. CPMG sequence is a special kind of SE sequence, so the formula can be preliminary expressed as follows.

$$
S=M_{0} \times \cos \omega t \times e^{-\frac{|t|}{T_{2} *}}
$$

Assuming $\eta$ represents the $\eta$ echo, so the time between the first echo and the $\eta$ echo can be expressed as follows.

$$
T(1, \eta)=\tau \times(1-\eta)+\frac{\tau}{2}
$$

Set $\mathrm{M}_{0}$ equals to 1000 , so the peak value of the $\eta$ echo can be expressed as follows.

$$
\operatorname{Peak}(1, \eta)=1000 \times e^{-\frac{|T(1, \eta)|}{T_{2}}}
$$

Each echo is attenuated in an exponential form, so the formula can be expressed as follows.

$$
S=\operatorname{Peak}(1, \eta) \times \cos \omega t \times e^{-\frac{|t|}{T_{2} *}}
$$

Therefore, the formula of CPMG sequence can be expressed as follows.

$$
S=1000 \times e^{-\frac{|T(1, \eta)|}{T_{2}}} \times \cos \left[0.001 \times\left(\gamma \times B_{0}-f\right) \times t\right] \times e^{-\frac{|t|}{T_{2}^{*}}}
$$

\section{SOFTWARE SIMULATION}

The interface of this system is designed by MATLAB GUI, and the initial interface is shown in figure 1. On the top of the interface is display area. On the bottom left is parameter adjustment area, and on the bottom right are three sequence options (Single-Pulse (SP) sequence, SE sequence, CPMG sequence). 


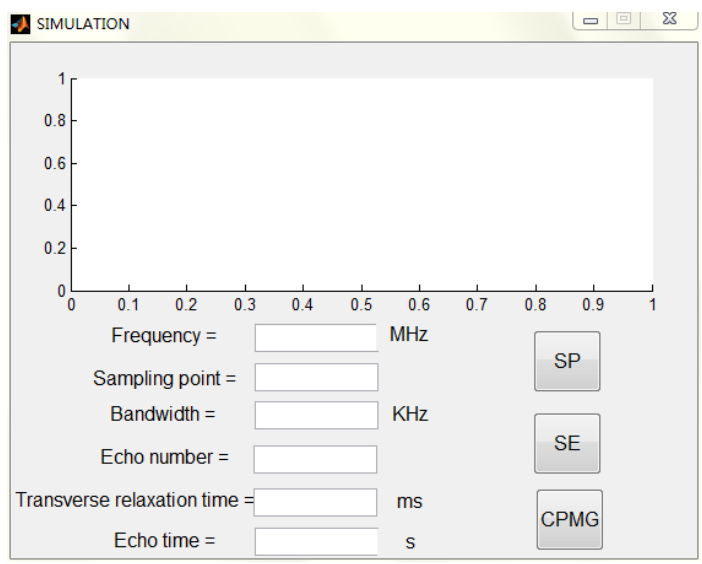

Figure. 1 GUI operating interface

\section{A. Single-Pulse Signal Simulation}

The parameters of the FID sequence are set as follows. The detection object is the hydrogen nucleus, and its magnetic field ratio is $42.58 \mathrm{MHz} / \mathrm{T}$. The main magnetic field strength is $0.5 \mathrm{~T}$. The transverse relaxation time is $10 \mathrm{~ms}$. The sampling bandwidth is $100 \mathrm{kHz}$. The signal sampling point is 4096. Larmor frequency in this case is $21.29 \mathrm{MHz}$, in which can achieve complete resonance state. Set RF frequency to be $21.289 \mathrm{MHz}$ to achieve off-resonance states, and the signal waveforms are as follows.

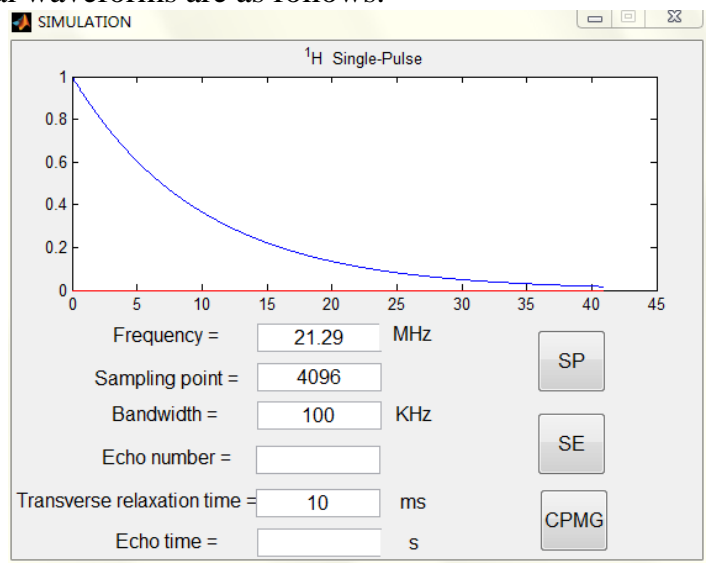

Figure. 2 FID signal waveform of hydrogen nuclei in resonance state

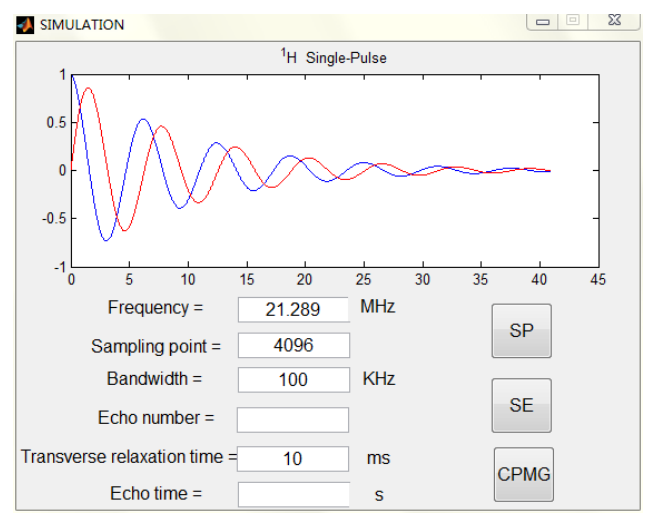

Figure.3 FID signal waveform of hydrogen nuclei in off-resonance state
Adjusting signal sampling point and the bandwidth, and other acquisition parameters to observe these impacts on the FID signal acquisition time.

- Changing signal sampling point, and the FID signal waveforms are as follows.

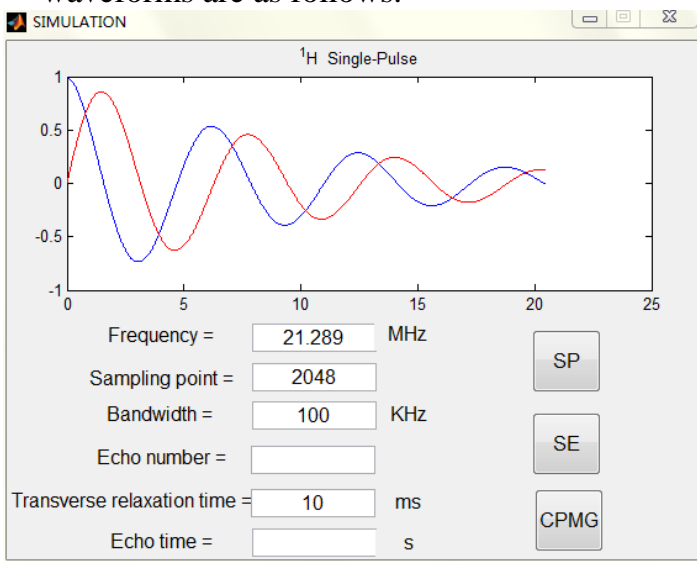

Figure. 4 FID signal $($ Sampling point $=2048)$

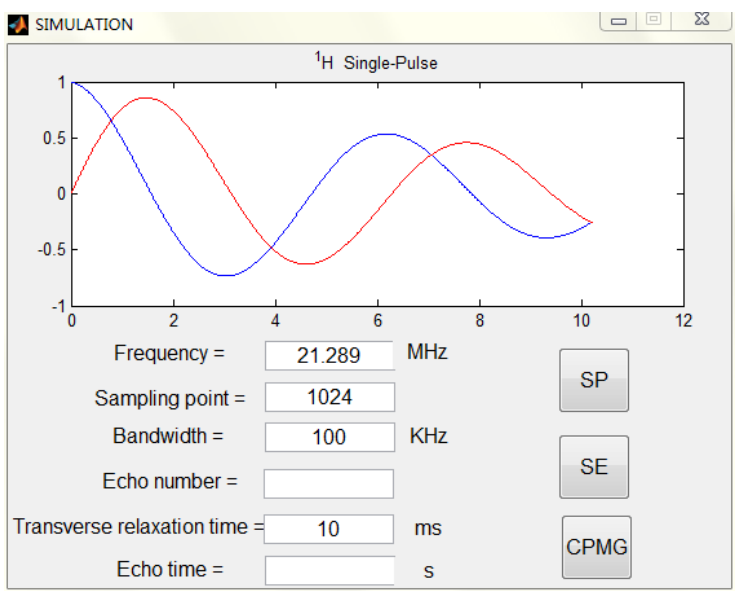

Figure. 5 FID signal $($ Sampling point $=1024)$

- Changing the sampling bandwidth, and the FID signal waveforms are as follows.

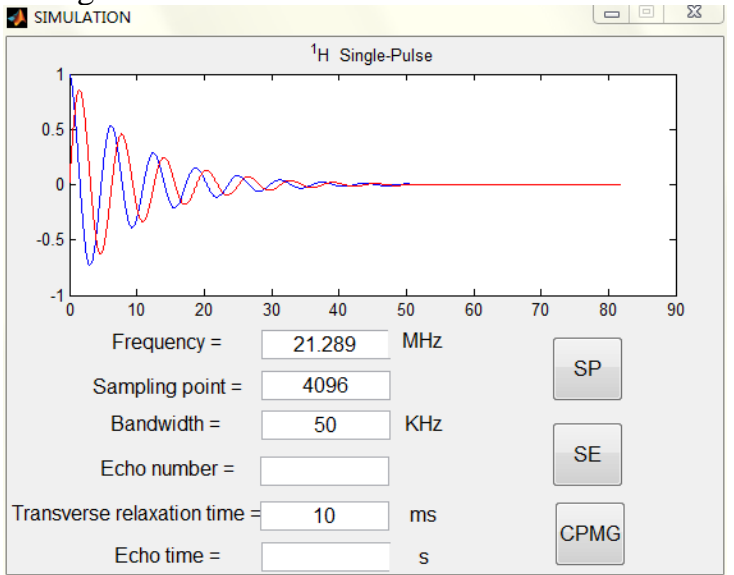

Figure. 6 FID signal (Bandwidth $=50 \mathrm{kHz}$ ) 


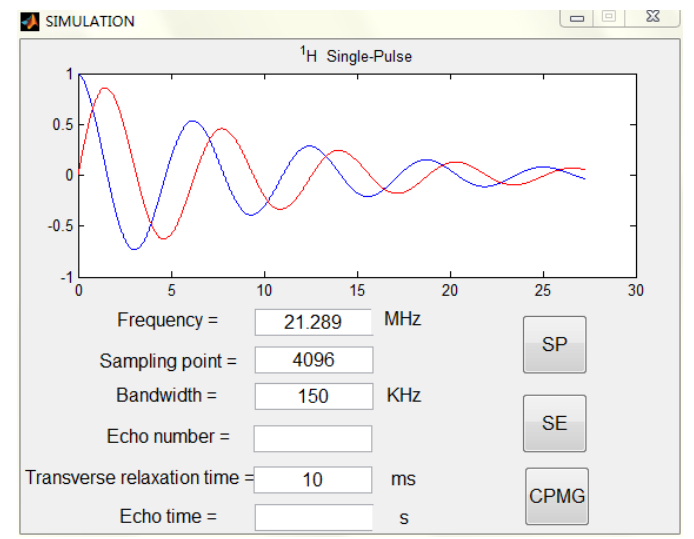

Figure. 7 FID signal $($ Bandwidth $=150 \mathrm{kHz})$

Comparing the results in figure 3, figure 4 and figure 5, the conclusion is the greater value of the sampling point, the longer acquisition time, when other parameters remain unchanged; and in figure 3, figure 6 and figure 7, the conclusion is the smaller bandwidth, the longer acquisition time.

\section{B. SE signal Simulation}

The parameters of the SE sequence are set as follows. The detection object is the hydrogen nucleus, and its magnetic field ratio is $42.58 \mathrm{MHz} / \mathrm{T}$. The main magnetic field strength is $0.5 \mathrm{~T}$. The transverse relaxation time is $10 \mathrm{~ms}$. The sampling bandwidth is $100 \mathrm{kHz}$. The signal sampling point is 4096. Larmor frequency in this case is $21.29 \mathrm{MHz}$, in which can achieve complete resonance state. Set RF frequency to be $21.289 \mathrm{MHz}$ to achieve off-resonance states, and the SE waveforms are as follows.

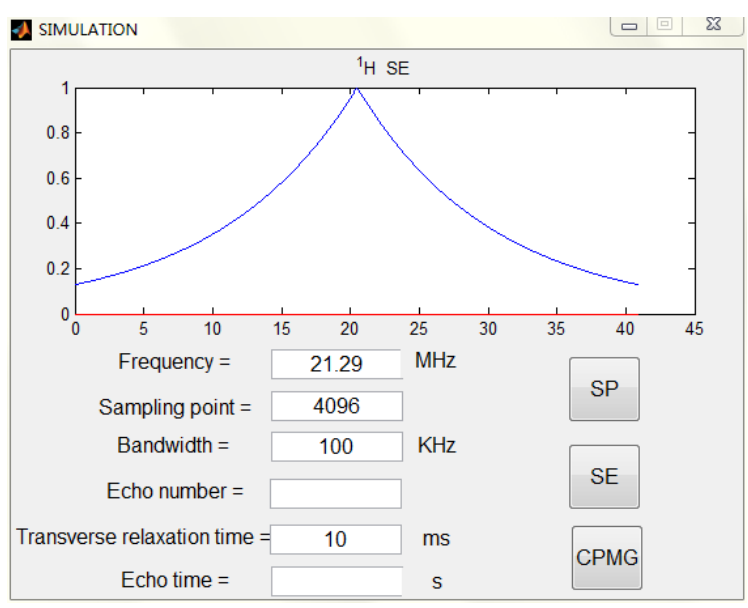

Figure. 8. SE signal waveform of hydrogen nuclei in resonance state.

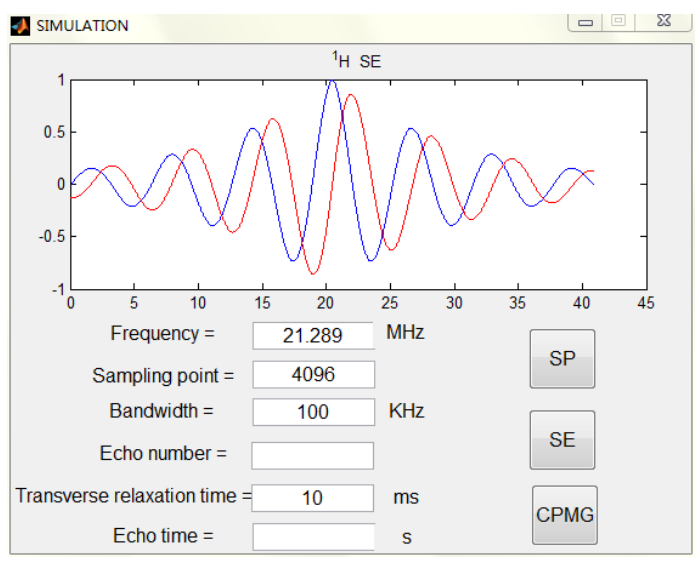

Figure 9. SE signal waveform of hydrogen nuclei in off-resonance state.

\section{CPMG signal simulation}

The parameters of the CPMG sequence are set as follows. The detection object is the hydrogen nucleus, and its magnetic field ratio is $42.58 \mathrm{MHz} / \mathrm{T}$. The main magnetic field strength is $0.5 \mathrm{~T}$. The transverse relaxation time is $10 \mathrm{~ms}$. The sampling bandwidth is $100 \mathrm{kHz}$. The signal sampling point is 4096. The echo time is 1 second. The time of $T_{2}^{*}$ is $0.1 \mathrm{~s}$. The echo number is 10 . Larmor frequency in this case is $21.29 \mathrm{MHz}$, in which can achieve complete resonance state. Set RF frequency to be $21.28 \mathrm{MHz}$ to achieve off-resonance states, and the CPMG waveforms are as follows.

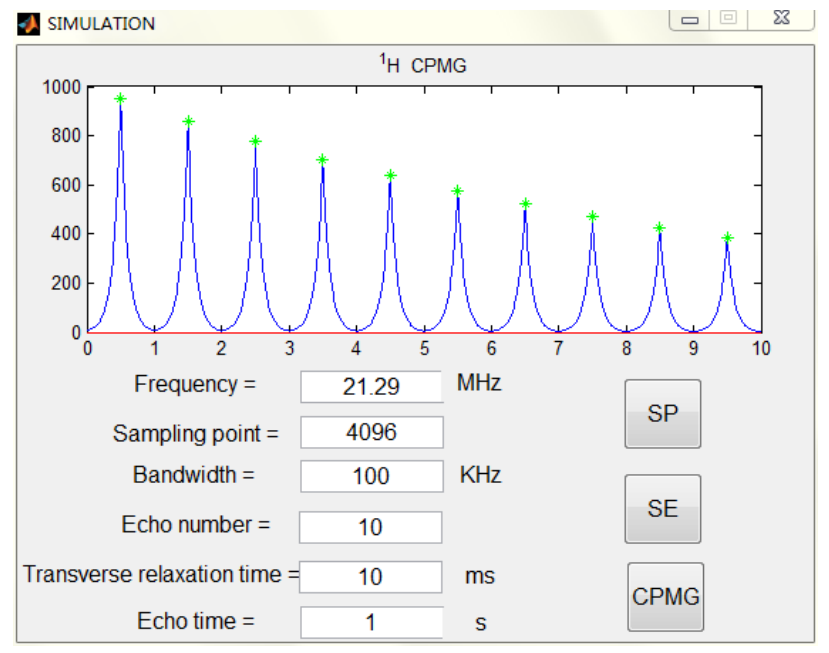

Figure 10.CPMG signal waveform of hydrogen nuclei in resonance state 


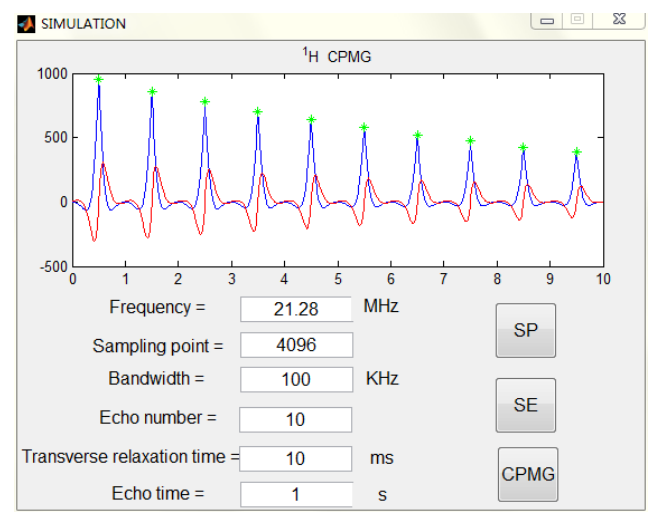

Figure 11. CPMG signal waveform of hydrogen nuclei in off-resonance state.

- Changing the transverse relaxation time, and CPMG signal waveform is as follows.

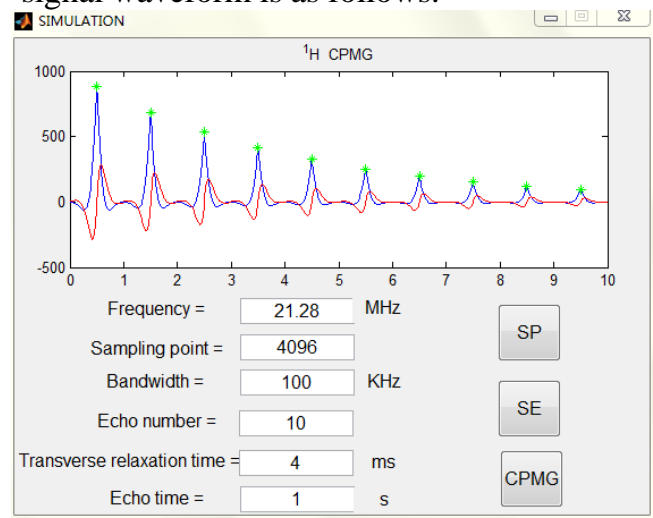

Figure 12. CPMG signal (Transverse relaxation time $=4 \mathrm{~ms}$ ).

- Changing the echo number, and CPMG signal waveform is as follows.

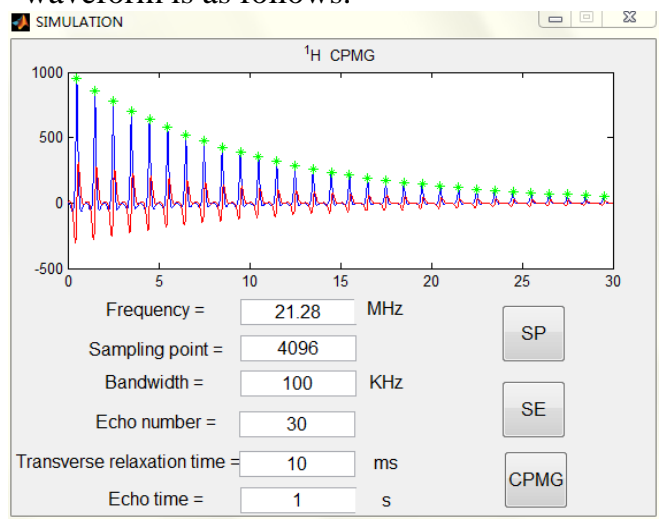

Figure 13. CPMG signal $($ Echo number $=30)$
Comparing the results in figure 11 and figure 12, the conclusion is the shorter transverse relaxation time is, the faster the echo signal attenuates; and in figure 11 and figure 13 , the conclusion is the more the number of echoes, the more obvious the gradually decreasing trend of echo peak of the CPMG sequence.

\section{CONCLUSION}

Based on MRI technique and theory of basic pulse sequence, an MR signal simulation system with adjustable parameter designed by MATLAB is proposed in this thesis. This system can realize the signal simulation of three kinds of sequences (Single-Pulse, SE, CPMG) and the main acquisition parameters can be changed to observe the experimental results, which not just helps to deepen the understanding of the principle of MRI, but can save a lot of scientific research time. This virtual system shall be also extended into other sequence, such as the gradient echo sequence. And other signal processing technique as the weight function of the original data processing can be added, which can make this system has strong practicability and better training for teaching and scientific research.

\section{ACKNOWLEDGMENT}

The paper is supported by the Key Curriculum Construction Fund of Shanghai Municipal Education Commission (Magnetic Resonance and Nuclear Medicine Imaging Equipments); the Minimally Invasive Inspirational Innovation Fund of USST.

\section{REFERENCES}

[1] Dabek J, Nieminen J O, Vesanen P T, et al. "Improved determination of FID signal parameters in low-field NMR,” Journal of Magnetic Resonance, jul, 2010, pp.148-160.

[2] Du Shimin, Yang Runping. "Development of teaching simulation platform of Signals and Systems based on Matlab GUI," Experimental Technology and Management, Vol. 29, No. 3, Mar.2012, pp.87-90. DOI: 10.3969/j.issn.1002-4956.2012.03.026.

[3] Wang Hong, Yang Kun, Liu Shuang, et al. "Analysis of continuous wave nuclear magnetic resonance signal," Journal of Hebei University (Natural Science Edition), Vol. 35, No. 6, 2015, pp.639643. DOI:10.3969/j.issn.1000-1565.2015.06.014.

[4] FU Yuan, CHU Zhen-dong "Signal Source and Primary Power Amplifier of Low Field NMR System," Instrument Technique and Sensor, No. 2, 2014, pp.102-104. DOI: 10.3969/j.issn.10021841.2014.02.033

[5] Chen Chen, Cao Weimin, Li Fei, et al. "Design and application of data acquisition system based on virtual technology," Computers and Applied Chemistry, Vol. 28, No. 12, December, 2011, pp.1553-1556. DOI: 10.3969/j.issn.1001-4160.2011.12.015. 